Virginia Commonwealth University VCU Scholars Compass

2007

\title{
Ground state structures and photoelectron spectroscopy of [Co-m(coronene) $](-)$ complexes
}

Anil K. Kandalam

Virginia Commonwealth University, akkandalam@vcu.edu

Boggavarapu Kiran

Virginia Commonwealth University

Puru Jena

Virginia Commonwealth University, pjena@vcu.edu

See next page for additional authors

Follow this and additional works at: http://scholarscompass.vcu.edu/phys_pubs

Part of the Physics Commons

Kandalam, A. K., Kiran, B., Jena, P., et al. Ground state structures and photoelectron spectroscopy of [Co$\mathrm{m}$ (coronene)](-) complexes. The Journal of Chemical Physics 126, 084306 (2007). Copyright (C) 2007 AIP LLC.

\section{Downloaded from}

http://scholarscompass.vcu.edu/phys_pubs/188

This Article is brought to you for free and open access by the Dept. of Physics at VCU Scholars Compass. It has been accepted for inclusion in Physics Publications by an authorized administrator of VCU Scholars Compass. For more information, please contact libcompass@vcu.edu. 
Authors

Anil K. Kandalam, Boggavarapu Kiran, Puru Jena, Xiang Li, Andrej Grubisic, and Kit H. Bowen 


\title{
Ground state structures and photoelectron spectroscopy of $\left[\mathrm{Co}_{m}\right.$ (coronene $\left.)\right]^{-}$complexes
}

\author{
Anil K. Kandalam, ${ }^{\text {a),b) }}$ Boggavarapu Kiran, and Puru Jena \\ Physics Department, Virginia Commonwealth University, Richmond, Virginia 23284 \\ Xiang Li, Andrej Grubisic, and Kit H. Bowen ${ }^{\mathrm{a}), \mathrm{c})}$ \\ Department of Chemistry, Johns Hopkins University, Baltimore, Maryland 21218 \\ and Department of Materials Science, Johns Hopkins University, Baltimore, Maryland 21218
}

(Received 13 November 2006; accepted 5 January 2007; published online 23 February 2007)

\begin{abstract}
A synergistic approach involving theory and experiment has been used to study the structure and properties of neutral and negatively charged cobalt-coronene $\left[\mathrm{Co}_{m}\right.$ (coronene)] complexes. The calculations are based on density functional theory with generalized gradient approximation for exchange and correlation potential, while the experiments are carried out using photoelectron spectroscopy of mass selected anions. The authors show that the geometries of neutral and anionic $\mathrm{Co}$ (coronene) and $\mathrm{Co}_{2}$ (coronene) are different from those of the corresponding iron-coronene complexes and that both the Co atom and the dimer prefer to occupy $\eta^{2}$-bridge binding sites. However, the magnetic coupling between the Co atoms remains ferromagnetic as it is between iron atoms supported on a coronene molecule. The accuracy of the theoretical results is established by comparing the calculated vertical detachment energies, and adiabatic electron affinities with their experimental data. (C) 2007 American Institute of Physics. [DOI: 10.1063/1.2437202]
\end{abstract}

\section{INTRODUCTION}

The interaction of transition metal atoms and metal clusters with hydrocarbons has been the subject of experimental and theoretical studies for many years. The availability of new experimental techniques to synthesize these complexes in the gas phase and the potential applications of these gasphase metal-organic complexes as building blocks of novel magnetic materials have renewed interest in this area recently. Several experimental ${ }^{1-7}$ and theoretical works ${ }^{3,4,8,9}$ have been devoted to studying the geometries and the electronic structures of transition metal atoms and clusters supported on benzene molecules. Transition metal-benzene complexes formed two distinct varieties of structures based on the transition metal involved. For the early-transition metal atoms $(\mathrm{Sc}-\mathrm{V})$, it was found that the benzene molecules sandwich the metal atoms to form sandwich and multidecker structures; ${ }^{1}$ while, for late-transition metals $(\mathrm{Fe}-\mathrm{Ni})$, the benzene molecules cage the transition metal atoms forming riceball structures. ${ }^{1,8}$ The rich structural variety and high stability exhibited by the transition metal-benzene complexes have further enhanced interest in metal-organic complexes.

Coronene $\left(\mathrm{C}_{24} \mathrm{H}_{12}\right)$, a polycyclic-aromatic hydrocarbon $(\mathrm{PAH})$, with six benzene rings fused to a central six-member ring, is a good prototype to study the interaction of metal clusters with extended systems such as graphite and large diameter carbon nanotubes. A coronene molecule, with its larger surface area, can bind multiple metal atoms or metal clusters more effectively than benzene, and thus, it may lead to a variety of metal-coronene multidecker complexes.

\footnotetext{
${ }^{\text {a) }}$ Authors to whom correspondence should be addressed.

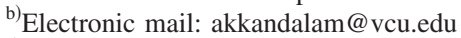

${ }^{c)}$ Electronic mail: kbowen@jhu.edu
}

Moreover, the presence of metal-PAH complexes has been identified as the possible source of infrared bands in the interstellar dust. ${ }^{10}$ Thus, owing to the possibility of diverse metal-coronene complexes and their rich metal- $\pi$ chemistry, these complexes have recently become the focus of several experimental $^{11-14}$ and theoretical studies. ${ }^{15,16}$

In one of the earliest gas-phase experimental studies ${ }^{11}$ on metal-coronene complexes, Dunbar and co-workers studied the interaction of metal cations such as $\mathrm{Mg}^{+}, \mathrm{Al}^{+}, \mathrm{Si}^{+}, \mathrm{In}^{+}$, $\mathrm{Pb}^{+}$, and $\mathrm{Bi}^{+}$with coronene molecules. In that work, the interaction of transition metal (TM) cations such as $\mathrm{Sc}^{+}$and $\mathrm{Mn}^{+}$with one or more coronene molecules was also studied. It was reported that the TM-coronene cationic complexes reacted readily and formed $\mathrm{TM}$ (coronene) $)_{2}$ complexes. Based on the rates of formation of the TM-coronene complexes, the lower limit on the binding energies of these complexes was estimated to be $32 \mathrm{kcal} \mathrm{mol}^{-1}$. Buchanan et al. ${ }^{12}$ have generated positively charged $\mathrm{Fe}_{m}$ (coronene) ${ }_{n}$ $(m=1-3, n=1,2)$ complexes, by laser vaporizing a composite sample in a pulsed nozzle cluster source. Based on photofragmentation studies, they reported that the iron binds to coronene as separate atoms and can form stable sandwich structures. However, a complementary theoretical study ${ }^{16}$ on $\mathrm{Fe}_{m}$ (coronene) $(m=1,2)$ complexes provided a different view of the structures. In that density functional theory (DFT) based study on neutral and cationic $\mathrm{Fe}_{m}$ (coronene) complexes, it was shown that $\mathrm{Fe}_{2}$ dimerizes and forms a strong bond with coronene. In another experimental study ${ }^{13}$ on metal-PAH complexes, Duncan et al., generated positively charged $\mathrm{Cr}_{m}$ (coronene $)_{n} \quad(m=1-5, n=1-3)$ complexes. The photofragmentation studies of these complexes provided evidence for the possibility of multimetal sandwich and multidecker sandwich structures. One of the most impor- 
tant observations of this study was that, depending on the experimental growth conditions, metal inserted $\mathrm{Cr}_{m}$ (coronene $)_{n}$ complexes can be produced. Duncan et al. ${ }^{14}$ reported anion photoelectron spectroscopic data on transition metal-coronene complexes, in which $\left.\left[\mathrm{V}_{m} \text { (coronene }\right)_{n}\right]^{-}$and $\left[\mathrm{Ti}_{m}(\text { coronene })_{n}\right]^{-} \quad(m=1-5, n=1-3)$ anionic complexes were produced by using laser vaporization techniques. Mass spectra of these complexes revealed that a maximum of five vanadium atoms can bind to a single coronene molecule though the mass peak intensities fall off gradually with increasing number of metal atoms. In the previously reported experimental study ${ }^{12}$ on positively charged $\mathrm{Fe}_{m}$ (coronene) complexes, no more than three iron atoms had been found to be bound to coronene molecule. Based on the photoelectron spectrum $^{14}$ and electron affinity (EA) values of these complexes, the authors made several observations: (1) The measured EA values of these complexes were greater than those of the corresponding free coronene molecule and pure metal clusters. (2) Depending on the experimental cluster source conditions, the $\left[\mathrm{V}(\text { coronene })_{2}\right]^{-}$anionic complex exhibited two different spectra, thus suggesting two possible structural configurations. (3) It was suggested that the onset of metal clustering on coronene surface depends on the number of metal atoms, and it was predicted that more than three metal atoms interacting with coronene can lead to metal clustering on the coronene surface. (4) $\left[\mathrm{Ti}(\text { coronene })_{2}\right]^{-}$does not form sandwich structures, but forms coronene dimer with metal atom binding externally to one of the coronene molecules. Even though several interesting conclusions were drawn about the geometries and electronic structures of metal and multi-metal-coronene complexes, no direct structural information on these complexes can be obtained from these experiments. However, theory and experiments together can provide insight into geometries, electronic structures, and magnetic properties of metal-coronene complexes, which neither alone can reliably supply.

In spite of considerable experimental focus on the metalcoronene systems and the resultant exciting findings, theoretical studies on metal-coronene complexes are scarce. Motivated both by this imbalance and the absence of any studies combining both experimental and theoretical efforts on these complexes, we have initiated a systematic experimental and theoretical study on $\mathrm{Co}_{m}$ (coronene) $(m=1-2)$ complexes. To our knowledge, neither experimental nor theoretical studies of anionic $\mathrm{Co}$ (coronene) complexes exist in the literature. Here, we report the results of our joint photoelectron spectroscopic and theoretical investigation of $\mathrm{Co}_{m}$ (coronene) complexes, focusing on the ground state geometric and electronic structures, vertical detachment energies, and magnetic properties. This combined effort has elucidated the unique bonding and structural features of $\mathrm{Co}_{m}$ (coronene) complexes. In addition, this study has also allowed us to assess the similarities and differences between $\mathrm{Co}_{m}$ (coronene) and the previously reported ${ }^{16}$ ground state geometries of $\mathrm{Fe}_{m}$ (coronene) complexes.

\section{METHODS}

\section{A. Experimental method}

Anion photoelectron spectroscopy is conducted by crossing a beam of mass-selected negative ions with a fixedfrequency photon beam and energy-analyzing the resultant photodetached electrons. The photodetachment process is governed by the energy-conserving relationship, $h \nu=\mathrm{EBE}$ $+\mathrm{EKE}$, where $h \nu$ is the photon energy, EBE is the electron binding energy, and EKE is the electron kinetic energy and by the selection rule that the multiplicity of the anion must change by \pm 1 as a result of photodetachment. These experiments were conducted on an apparatus consisting of a laser vaporization source, a linear time-of-flight mass selector, a Nd:YAG (yttrium aluminum garnet) photodetachment laser, and a magnetic bottle electron energy analyzer. Our apparatus has been described previously. ${ }^{5}$ The anion complexes of interest were generated by condensing coronene vapor onto a rotating, translating cobalt target rod prior to laser vaporization. Helium from a pulsed valve was used as the expansion gas.

\section{B. Computational method}

The electronic structure calculations of neutral and negatively charged $\mathrm{Co}_{m}$ (coronene) complexes were carried out within the framework of generalized gradient approximation to DFT using GAUSSIAN03 code. ${ }^{17}$ We have employed gradient corrected Becke's exchange ${ }^{18}$ and Perdew-Wang correlation $^{19}$ functionals (BPW91) in these calculations. The triple- $\zeta$ basis sets $\left(6-311 \mathrm{G}^{* *}\right)$ and the frozen-core Lan $12 \mathrm{dz}$ basis sets were used for coronene and cobalt, respectively. The reliability and the accuracy of the functional form and the basis sets have been established in our previous studies ${ }^{9}$ on $\mathrm{V}_{m}$ (benzene) ${ }_{n}$ complexes. The coronene molecule, because of its polycyclic character, offers a wide variety of binding sites for the metal atom, viz., site on top of central/ peripheral rings $\left(\eta^{6}\right)$ and various $\mathrm{C}-\mathrm{C}$ bridge sites $\left(\eta^{2}\right)$ between the central and peripheral rings, between two peripheral rings, and on the edge of the peripheral ring. Hence, in order to identify the ground state geometry of the $\mathrm{Co}_{m}$ (coronene) complex, we have taken into account all of these structural configurations during the geometry optimization process. In addition, for each of the structural configurations, various possible spin multiplicities were also considered. The geometry optimization calculations were performed without any symmetry constraints. Vibrational frequency calculations were performed to ascertain the stability of lowest energy isomers. Not all the higher energy isomers are presented in this paper. However, the relative energies and structural parameters of the higher energy isomers can be obtained from the authors.

The vertical detachment energies (VDEs) were calculated following the definition $\mathrm{VDE}=E_{2}-E_{1}$, where $E_{1}$ is the total energy of the anion and $E_{2}$ is the total energy of the neutral, both calculated at the anion's ground state geometry. For the anionic complex with multiplicity $M$, neutral species with multiplicities $M-1$ and $M+1$ were considered in the VDE calculation. The higher transition energies were calculated by following the extended Koopmans' theorem, ${ }^{20}$ in 


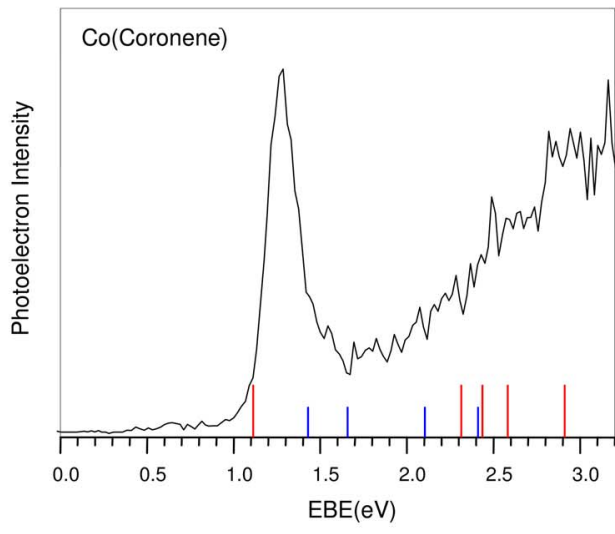

(a)

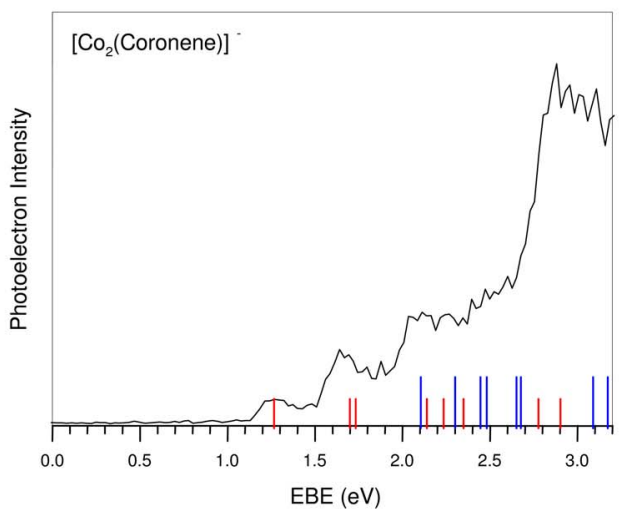

(b)

FIG. 1. (Color online) Photoelectron spectra of (a) $[\mathrm{Co}(\text { coronene })]^{-}$and (b) $\left[\mathrm{Co}_{2} \text { (coronene) }\right]^{-}$. The vertical lines indicate the calculated transitions. In $\left[\mathrm{Co}(\text { coronene) }]^{-}\right.$, the red (longer) lines indicate the transitions from triplet to doublet, while the blue (shorter) lines indicate the transitions from triplet to quartet. In $\left[\mathrm{Co}_{2} \text { (coronene) }\right]^{-}$, the red (shorter) lines and blue (longer) lines represent transitions from quarter to quintet and triplet, respectively.

which a correction term $\delta E$, was added to the eigenvalues of the ground state anion. The correction term $\delta E$ is defined by the equation $\delta E=E_{1}-E_{2}-\varepsilon_{\mathrm{HOMO}}$, where $E_{1}$ and $E_{2}$ are the same as discussed above, and $\varepsilon_{\mathrm{HOMO}}$ corresponds to the eigenvalue of the highest occupied molecular orbital (HOMO) of the anion in its ground state.

\section{RESULTS AND DISCUSSION}

\section{A. Photoelectron spectra}

The photoelectron spectra of $[\mathrm{Co}(\text { coronene })]^{-}$and $\left[\mathrm{Co}_{2} \text { (coronene) }\right]^{-}$anions are presented in Fig. 1. Both spectra were recorded with $3.49 \mathrm{eV}$ photons (355 nm light) from the third harmonic of a Nd:YAG laser. The photoelectron spectrum of $[\mathrm{Co}(\text { coronene })]^{-}$is dominated by a single relatively sharp peak centered at $1.31 \mathrm{eV}$ with unresolved vibronic structure gradually rising to its high electron binding energy side. The photoelectron spectrum of $\left[\mathrm{Co}_{2} \text { (coronene) }\right]^{-}$exhibits four, or perhaps five, resolved peaks, with the lowest electron binding energy peak centered at $1.28 \mathrm{eV}$ and with each successive peak to its high EBE side increasing in intensity.

\section{B. Computed geometries}

The Co(coronene) complex (anions and neutrals). In this system, the binding sites available for the Co atom/anion on the surface of coronene are the on-top sites $\left(\eta^{6}\right)$ above the central ring and peripheral rings, and various $\mathrm{C}-\mathrm{C}$ bridge sites $\left(\eta^{2}\right)$. The ground state geometry and the next higher energy structure of $[\mathrm{Co}(\text { coronene })]^{-}$anionic complexes are given in Fig. 2. Our calculations revealed that in the $[\mathrm{Co}$ (coronene $)]^{-}$, the $\mathrm{Co}^{-}$anion prefers to occupy the $\mathrm{C}-\mathrm{C}$ $\eta^{2}$-bridge site on the peripheral ring of coronene. The triplet spin state $(2 S+1=3)$ is the most preferred spin state in the anionic system. The geometry corresponding to cobalt occupying the $\eta^{6}$-on-top site of the peripheral ring is $0.25 \mathrm{eV}$ higher in energy and prefers the singlet $(2 S+1=1)$ spin state. If the spin state of this $\eta^{6}$ geometry is restricted to the triplet $(2 S+1=3)$ during the geometry optimization process, the cobalt atom moves away from the $\eta^{6}$-on-top site to the $\eta^{2}$-bridge site.
The preference for the $\eta^{2}$ over the $\eta^{6}$ binding site in the anionic complex can be explained with the help of molecular orbital pictures for these two complexes. Upon molecular orbital (MO) analysis, it was found that the lowest unoccupied molecular orbital (LUMO) in the neutral $\eta^{6}$ structure is mainly concentrated on the metal atom, with a small antibonding contribution from metal-carbon interaction, whereas the LUMO of the neutral $\eta^{2}$ structure is a nonbonding orbital located on metal atom. Thus, in the anion the $\eta^{2}$ binding site on the peripheral ring is the more preferred site for cobalt. The nonbonding character of the HOMO of ground state geometry of $[\mathrm{Co}$ (coronene $)]^{-}$is discussed in the next section.

Unlike $[\mathrm{Co}(\text { coronene })]^{-}$, in the neutral complex the cobalt atom prefers to occupy the $\eta^{6}$ site of the peripheral ring. However, the structure where the cobalt atom binds to the $\mathrm{C}-\mathrm{C} \eta^{2}$ site of the peripheral ring is energetically degenerate $(\Delta E=0.02 \mathrm{eV})$ with the $\eta^{6}$ binding site. The two lowest energy isomers are shown in Fig. 3. The doublet $(2 S+1=2)$ spin state is found to be the ground state spin multiplicity of the neutral complex.

It is interesting to note that though the ground state geometry of neutral $\mathrm{Co}$ (coronene) is similar to the previously reported ${ }^{14}$ geometry of neutral $\mathrm{Fe}($ coronene), the stabilities of the higher energy isomers of these complexes are different. In the case of the $\mathrm{Fe}$ (coronene) complex, ${ }^{16}$ it was re-
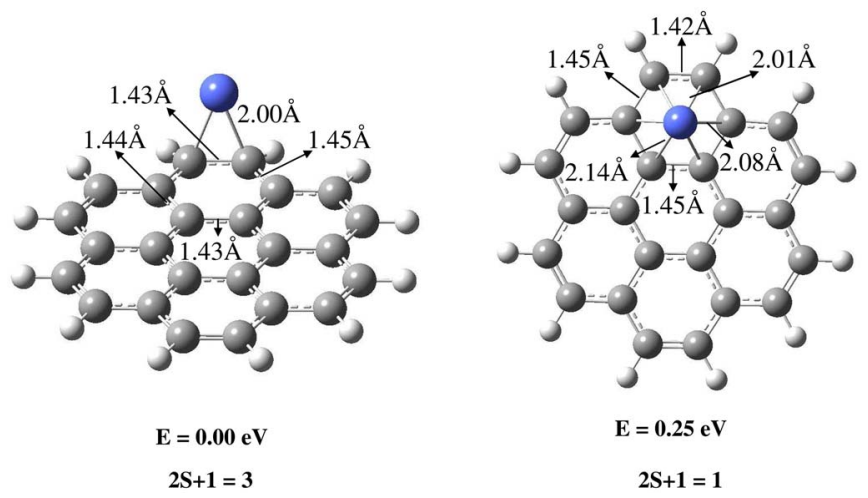

FIG. 2. (Color online) Ground state geometry and the next higher energy geometry of negatively charged $[\mathrm{Co}($ coronene $)]$ complex. 


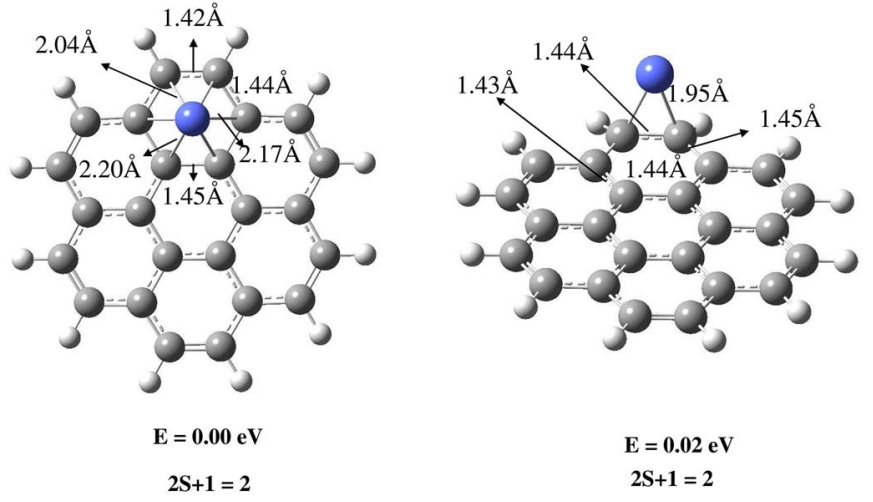

FIG. 3. (Color online) Ground state geometry and the next higher energy geometry of neutral $[\mathrm{Co}($ coronene $)]$ complex.

ported that the geometry corresponding to $\eta^{2}$ binding site was not even a local minima and the iron atom moved away from the bridge site towards the center of the ring; whereas in Co(coronene), the $\eta^{2}$ binding site of the peripheral ring is not only stable but also energetically degenerate with the $\eta^{6}$ binding site (see Fig. 3).

The $\mathrm{Co}_{2}$ (coronene) complex (anions and neutrals). We now present and discuss our results on the interaction of two cobalt atoms with the coronene molecule, in both anionic and neutral forms. In this system, we have taken into account various structural configurations, in which two cobalt atoms bind both associatively (dimerlike) and dissociatively (atomiclike) to various binding sites on the surface of a coronene molecule. Figure 4 shows the ground state geometry of the $\left[\mathrm{Co}_{2} \text { (coronene) }\right]^{-}$complex, along with other higher energy isomers. The ground state geometry [Fig. 4(a)] corresponds to a $\mathrm{Co}_{2}$ dimer occupying the $\eta^{2}$ site on the peripheral ring, with the dimer bond axis almost perpendicular to the surface of the coronene molecule ( $C_{s}$ symmetry). Thus, contrary to our intuition, the second cobalt atom does not interact with the coronene molecule thereby demonstrating the preference of $\mathrm{Co}-\mathrm{Co}$ bonding over Co-coronene bonding. The $\mathrm{Co}-\mathrm{Co}$ bond length in this configuration elongates to $2.26 \AA$ compared with the bond length in $\mathrm{Co}_{2}$ dimer $(2.15 \AA)$. The preference for the $\eta^{2}$ binding site over the $\eta^{6}$ site can be seen as an attempt to minimize the disruption of the aromatic nature of coronene molecule due to its interaction with metal atoms. The ground state spin multiplicity of the $\left[\mathrm{Co}_{2} \text { (coronene) }\right]^{-}$anionic complex is predicted to be a quartet $\left({ }^{4} A^{\prime}\right)$. There also exists a higher energy isomer $(\Delta E=0.20 \mathrm{eV})$ in which the two cobalt atoms dimerize, and both of the cobalt atoms are directly interacting with the coronene molecule [see Fig. 4(b)]. The energy difference between these two isomers [Figs. 4(a) and 4(b)] is within the uncertainty of our calculations. Thus, based on theoretical calculations alone, it is not possible to identify the correct ground state geometry of $\left[\mathrm{Co}_{2} \text { (coronene) }\right]^{-}$. However, comparison of the calculated VDEs and adiabatic electron affinity (AEA) of these two geometries with the measured photodetachment energies can distinguish between them and identify the correct ground state geometry. These aspects are discussed in the next section. The isomer in which the two cobalt atoms occupy the bridge sites of a peripheral ring [Fig. 4(c)] is found to be higher in energy by $0.40 \mathrm{eV}$ than the ground state geometry, while isomer with the cobalt atoms occupying the bridge sites of central ring [Fig. 4(d)] is $0.87 \mathrm{eV}$ higher in energy.

The geometries of ground state and higher energy isomers of neutral $\mathrm{Co}_{2}$ (coronene) are given in Fig. 5. The ground state geometry of neutral $\mathrm{Co}_{2}$ (coronene) complex [Fig. 5(a)] is predicted to be the same as that of the anionic complex with spin multiplicity $(2 S+1)=5$. However, when we compare the ground state geometry of the neutral $\mathrm{Co}_{2}$ (coronene) complex with that of previously reported $\mathrm{Fe}_{2}$ (coronene), we find that they are completely different from each other. In the $\mathrm{Fe}_{2}$ (coronene) study, ${ }^{16}$ it was reported that the iron atoms prefer to reside above the center of the
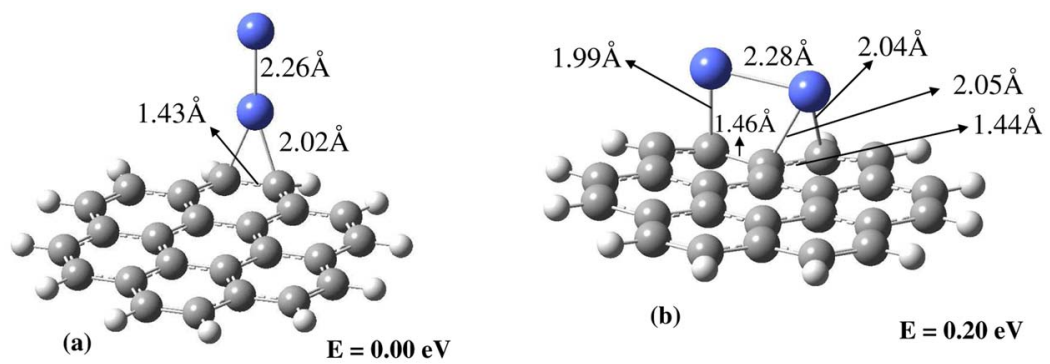

(b)

$\mathrm{E}=0.20 \mathrm{eV}$
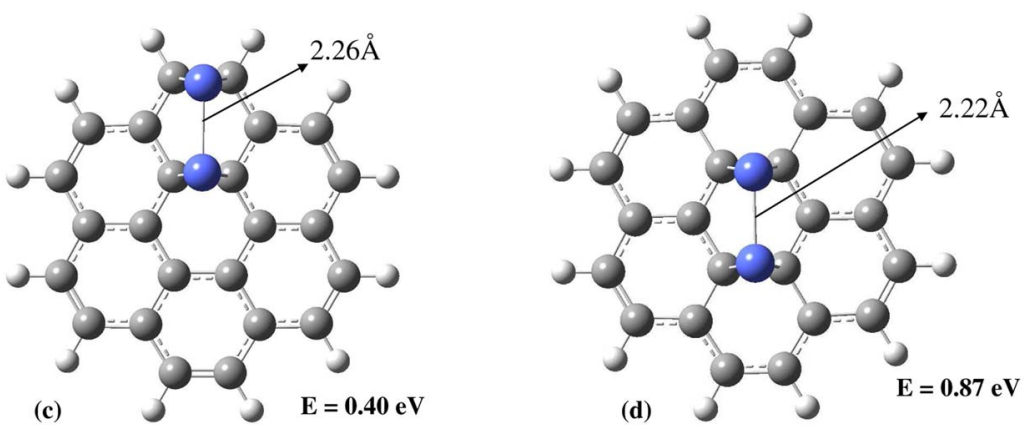

FIG. 4. (Color online) Ground state geometry and higher energy geometries of negatively charged $\left[\mathrm{Co}_{2}(\right.$ coronene $\left.)\right]$ complex. 

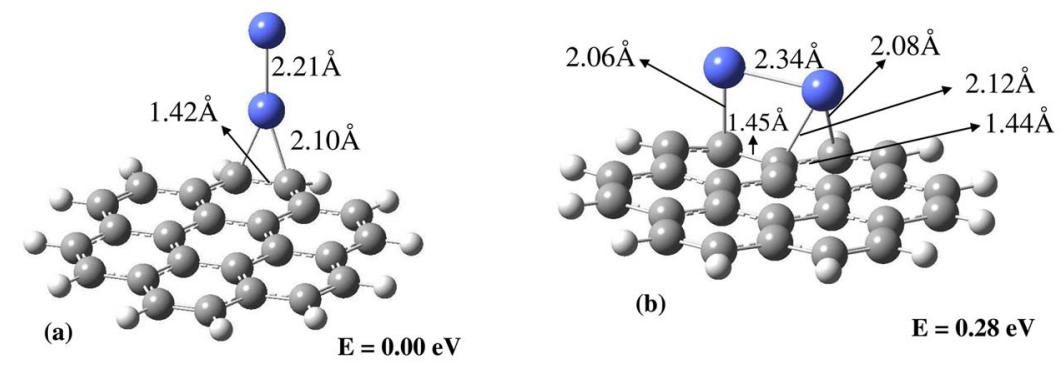

(b)
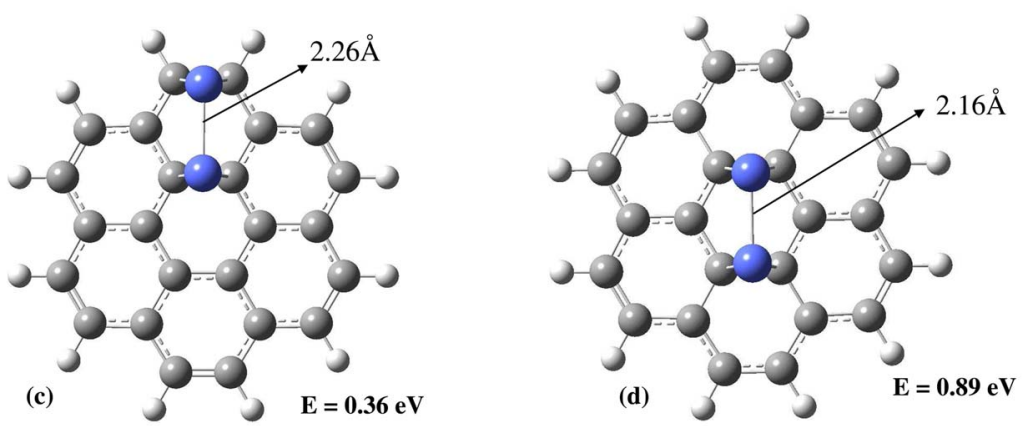

FIG. 5. (Color online) Ground state geometry and higher energy geometries of neutral $\left[\mathrm{Co}_{2}\right.$ (coronene) $]$ complex.

two adjacent peripheral rings. However, in the case of $\mathrm{Co}_{2}$ (coronene), this geometry does not even correspond to a local minimum. In fact, when cobalt atoms were placed at the $\eta$ site of the adjacent peripheral rings, they moved away from the center, with one cobalt atom occupying the C-C $\eta^{2}$ site and the second cobalt atom sitting directly above the peripheral carbon atom [see Fig. 5(b)]. This structure is higher in energy than the ground state geometry by $0.28 \mathrm{eV}$. For the $\mathrm{Fe}_{2}$ (coronene) complex, ${ }^{16}$ it was also reported that the ground state geometry is closely followed by another geometry $(\Delta E=0.16 \mathrm{eV})$ in which the two metal atoms bind over the bridge sites of the peripheral ring. In the $\mathrm{Co}_{2}$ (coronene) complex, however, this geometry [Fig. 5(c)] is $0.36 \mathrm{eV}$ higher in energy than the ground state geometry.

It was predicted earlier ${ }^{12}$ that iron prefers to bind as separate atoms with the coronene molecule and a minimum of four metal atoms ( $\mathrm{V}$ and $\mathrm{Ti}$ ) are required before clustering of metal atoms on the coronene ${ }^{14}$ can ensue. However, the current results along with previously reported ${ }^{16}$ $\mathrm{Fe}_{2}$ (coronene) theoretical results are not in agreement with these predictions. Thus we conclude that the preferential binding sites $\left(\eta^{2}\right.$ vs $\left.\eta^{6}\right)$, the onset of metal clustering, the orientation of the metal cluster, and its interaction with the coronene molecule are different for different metal atoms.

\section{Comparison of theoretical predictions and experimental spectra}

We now compare the calculated VDEs of the $[\mathrm{Co}(\text { coronene })]^{-}$and $\left[\mathrm{Co}_{2}(\text { coronene })\right]^{-}$anion complexes with their experimental photoelectron spectra. Good agreement would strongly suggest that our predicted ground state geometries and their corresponding spin multiplicities are correct.

As mentioned earlier, the ground state of the $[\mathrm{Co}(\text { coronene })]^{-}$anion is a triplet spin state. The VDEs corresponding to transitions from the anion's triplet to the neutral's doublet and quarter states are calculated to be 1.12 and $1.42 \mathrm{eV}$, respectively. The difference in the ground state electronic energies of $\mathrm{Co}($ coronene $)$ versus $[\mathrm{Co}(\text { coronene })]^{-}$ is reflected in the lowest EBE peak of the experimental spectrum of $[\mathrm{Co} \text { (coronene) }]^{-}$[see Fig. 1(a)]. The broadening observed in that peak, however, is probably not due to the small energy difference between the $\eta^{2}$ and $\eta^{6}$ binding sites in the neutral Co(coronene) complex. The broadening is more likely due to an overlap of transitions from the anion's ground state to the doublet and quartet states of the neutral. These transitions are predicted to occur at 1.12 and $1.42 \mathrm{eV}$ and thus would be located on the either side of this peak [see Fig. 1(a)]. The calculated AEA for the Co(coronene) complex is $1.06 \mathrm{eV}$, which is in excellent agreement with the experimental observation $(1.15 \pm 0.15 \mathrm{eV})$. The frontier molecular orbitals of $[\mathrm{Co}(\text { coronene })]^{-}$, from which the electron detachments occur, are shown in Fig. 6.

Lastly, it is interesting to compare the electron affinities of molecular coronene $(0.47$ and $0.54 \mathrm{eV}),{ }^{21,22}$ the cobalt atom $(0.66 \mathrm{eV}),{ }^{23}$ and the $\mathrm{Co}$ (coronene) complex $(1.15 \mathrm{eV})$. Both moieties in the $\mathrm{Co}$ (coronene) complex have positive adiabatic electron affinities with comparable values. In spite of this, however, our calculations indicate that the excess electron resides mostly on the cobalt moiety. This suggests an anion-molecule interaction between $\mathrm{Co}^{-}$and coronene, after all coronene is polarizible enough to provide the $0.49 \mathrm{eV}$ in solvation energy, i.e., $0.66 \mathrm{eV}+0.49 \mathrm{eV}$ $=1.15 \mathrm{eV}$. The problem with this, however, is that the $[\mathrm{Co}$ (coronene $)]^{-}$spectrum does not show the three peak pattern that would be characteristic of a $\mathrm{Co}^{-}$chromophore in an anion-molecule complex. Thus, we conclude that the interaction is significantly more complex.

The next set of higher energy photodetachment transitions is due to the electron detachments from $\beta$ orbitals (transition to neutral quartet) and as calculated to be $1.66,2.12$, and $2.42 \mathrm{eV}$; while the still higher energy transitions (to the neutral doublet state) occur at 2.32, 2.43, 2.59, and $2.91 \mathrm{eV}$. The calculated transitions are consistent with the experimental values [Fig. 1(a)]. It is to be noted here that the transitions with EBE values less than $2.50 \mathrm{eV}$ correspond to the electron 

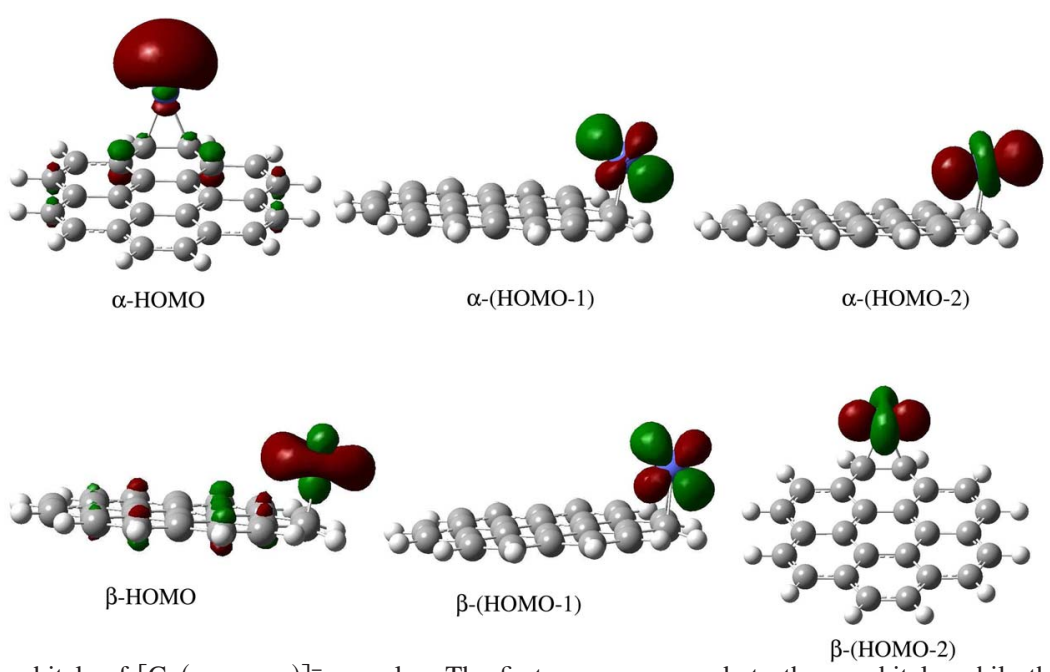

FIG. 6. (Color online) Frontier orbitals of $\left[\mathrm{Co}(\text { coronene) }]^{-}\right.$complex. The first row corresponds to the $\alpha$ orbitals, while the second row corresponds to $\beta$ orbitals.

detachments from nonbonding orbitals, mainly located on the metal atom (see Fig. 6), whereas the transitions with EBE values higher than $2.50 \mathrm{eV}$ correspond to electron detachment from bonding orbitals. The calculated detachment energies resulting in both doublet and quartet states, along with the AEA of [Co(coronene) $]$ system, are collected in Table I.

For $\left[\mathrm{Co}_{2} \text { (coronene) }\right]^{-}$, whose ground state spin multiplicity is a quartet, the transitions from the anion's quartet to the neutral's quintet and triplet were calculated. We first discuss the detachment energies from the lowest energy isomer of $\left[\mathrm{Co}_{2} \text { (coronene) }\right]^{-}$[see Fig. 4(a)]. The VDEs corresponding to the transitions from the anion's quartet state to the neutral's quintet and triplet states are 1.27 and $2.11 \mathrm{eV}$, respectively. All the calculated transition energies and AEA values for this complex are given in Table II. The first electron detachment, corresponding to resultant neutral quintet $(\mathrm{VDE}=1.27 \mathrm{eV})$, is not from the unpaired electron, but from $36 a^{\prime}$, a $\beta \mathrm{MO}$, which corresponds to $d_{z}^{2}$ antibonding orbital between the metal atoms [see Fig. 7(b)]. The calculated VDE value is in excellent agreement with the experimental value of $1.3 \pm 0.1 \mathrm{eV}$ [Fig. 1(b)]. The calculated AEA of this complex is $1.20 \mathrm{eV}$, which is also consistent with the spectrum $(1.15 \pm 0.1 \mathrm{eV})$. The small energy difference between the calculated VDE and AEA $(\Delta E=0.07 \mathrm{eV})$ is consistent with the fact that there is negligible structural change in the ground state geometry of $\mathrm{Co}_{2}$ (coronene) complex due to the removal of the extra electron. The next two electron detachments (transition to neutral quintet states) are also from the $\beta$-MOs,

TABLE I. The calculated vertical detachment energies (VDEs) and adiabatic electron affinity (AEA) for $[\mathrm{Co}(\text { coronene })]^{-}$complex. The energies are given in $\mathrm{eV}$.

\begin{tabular}{cccc}
\hline \hline System & $\begin{array}{c}\operatorname{VDE}(\alpha) \\
(\text { doublet })\end{array}$ & $\begin{array}{c}\operatorname{VDE}(\beta) \\
\text { (quartet) }\end{array}$ & AEA \\
\hline & 1.12 & 1.42 & 1.06 \\
(Co-coronene) $^{-}$ & 2.32 & 1.66 & \\
& 2.43 & 2.12 & \\
& 2.59 & 2.42 & \\
& 2.91 & & \\
\hline
\end{tabular}

i.e., $\beta-26 a^{\prime \prime}$ and $\beta-a^{\prime}$, which correspond to VDEs of 1.70 and $1.73 \mathrm{eV}$, respectively; while we observe a broad transition centered at $1.65 \mathrm{eV}$ [Fig. 1(b)]. Both the $26 a^{\prime \prime}$ and $35 a^{\prime}$ $\beta$-MOs correspond to nonbonding orbitals on the metal atoms [Fig. 7(b)]. The remaining transitions to the neutral quintet state were calculated to be 2.14 (from $\beta-34 a^{\prime}$ ), 2.24 (from $\beta-33 a^{\prime}$ ), 2.35 (from $\beta-32 a^{\prime}$ ), 2.79 (from $\beta-25 a^{\prime \prime}$ ), and $2.90 \mathrm{eV}$ (from $\beta-31 a^{\prime}$ ).

The first transition to the neutral triplet state (VDE $=2.11 \mathrm{eV})$ corresponds to detachment of an electron from $\alpha$-MO $\left(\alpha-37 a^{\prime}\right)$, which is again a $d_{z}^{2}$ orbital of antibonding nature between the metal atoms [see Fig. 7(a)]. This again is in good agreement with the experimental value of $2.1 \pm 0.1 \mathrm{eV}$. The next detachment is from $\alpha-36 a^{\prime} \mathrm{MO}$, which is the first unpaired electron [Fig. 7(a)], and the corresponding transition energy is calculated to be $2.30 \mathrm{eV}$. The third transition from $\alpha$-MO $\left(\alpha-28 a^{\prime \prime}\right)$ corresponds to an electron detachment from the nonbonding orbitals located on the metal atoms [Fig. 7(a)] and the resulting transition energy is $2.44 \mathrm{eV}$. The higher transition energies for transitions to the triplet neutral states were calculated to be 2.49 (from $\alpha-35 a^{\prime}$ ), 2.66 (from $\alpha-34 a^{\prime}$ ), 2.68 (from $\alpha-33 a^{\prime}$ ), 3.09 (from $\left.\alpha-27 a^{\prime \prime}\right)$, and $3.18 \mathrm{eV}$ (from $\left.\alpha-32 a^{\prime}\right)$.

As there exists an anionic isomer [Fig. 4(b)], close in energy $(\Delta E=0.20 \mathrm{eV})$ from the ground state isomer and within our computational uncertainty, we cannot rule out the

TABLE II. The calculated vertical detachment energies and adiabatic electron affinity $(\mathrm{AEA})$ of $\left[\mathrm{Co}_{2} \text { (coronene) }\right]^{-}$. The energies are given in $\mathrm{eV}$.

\begin{tabular}{cccc}
\hline \hline System & $\begin{array}{c}\operatorname{VDE}(\alpha) \\
(\text { doublet })\end{array}$ & $\begin{array}{c}\operatorname{vDE}(\beta) \\
\text { (quartet) }\end{array}$ & AEA \\
\hline (Co $_{2}$-coronene $)^{-}$ & 2.11 & 1.27 & 1.20 \\
& 2.30 & 1.70 & \\
& 2.44 & 1.73 & \\
2.49 & 2.14 & \\
2.66 & 2.24 & \\
& 2.68 & 2.35 & \\
& 3.09 & 2.79 & \\
& 3.18 & 2.90 & \\
\hline
\end{tabular}




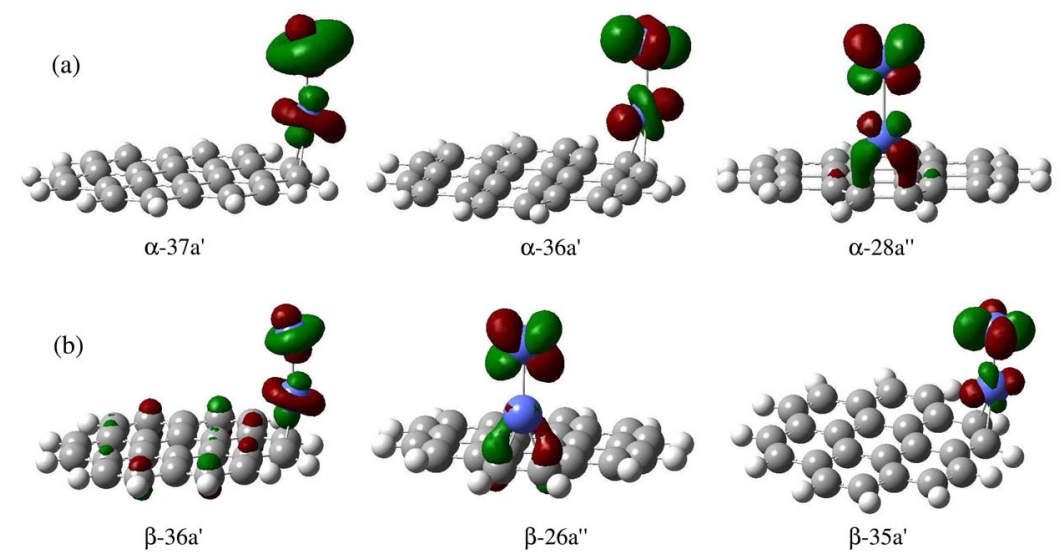

FIG. 7. (Color online) (a) Three valence $\alpha$-molecular orbitals (MOs) of $\left[\mathrm{Co}_{2}(\text { coronene) }]^{-}\right.$. (b) Three valence MOs corresponding to $\beta$ occupation.

possibility that this higher energy isomer may be contributing to the observed spectrum. To check for this possibility, we have calculated the two lowest transition energies and the adiabatic electron affinity for this isomer and compared them with the measured spectrum. The calculated transitions to the neutral triplet and quintet states were calculated to be 1.56 and $1.40 \mathrm{eV}$, respectively. The AEA for this complex was calculated to be $1.36 \mathrm{eV}$. As these values do not match observed photoelectron spectra we can conclude that it is the lowest energy isomer [Fig. 4(a)] rather than the second higher energy isomer [Fig. 4(b)] that is responsible for most of the observed transitions, although some contributions from the next higher energy isomer cannot be ruled out.

In the lower energy range $(<2.30 \mathrm{eV})$ of the experimental spectrum, the majority of the peaks are accounted for by the electron detachments from $\beta$-MOs, while the higher energy range is dominated by the detachments from $\alpha$-MOs. In addition, the low EBE, low intensity peaks in the highly structured part of the experimental PES spectrum [see Fig. 1(b)] are mainly due to the electron detachments from the metal atom lone pairs (nonbonding MOs), while the more intense peaks $(\mathrm{EBE}>2.7 \mathrm{eV})$ correspond to detachments from either the metal-metal or metal-carbon bonding orbitals.

When the predicted photoelectron spectra match well with the measured ones, one can be reasonably confident that the calculations have succeeded in determining the main features of the electronic states involved. Among the properties calculated are the spin multiplicities of these states, and the spin magnetic moment, $\mu_{n}$, of a state is equal to the spin multiplicity minus one, i.e., $(2 S+1)-1=2 S$ or $\mu_{n} / \mu_{B}=2 S$. Also, since orbital magnetic moments are usually small compared to spin magnetic moments in metals, the spin magnetic moment becomes a reasonable estimate of the total magnetic moment of a given metal-containing system. As a practical matter, the states that would be of most potential interest in nanoscience and nanotechnology are the ground states of the neutral complexes, because they are the ones that would best mirror real surface interactions between metal atom clusters and organic molecular substrates. In the present study, the ground state of neutral $\mathrm{Co}$ (coronene) is seen to have a spin magnetic moment of $1 \mu_{B}$. For comparison, the spin magnetic moment for a free cobalt atom is $3.0 \mu_{B}$, while it is $1.7 \mu_{B} /$ at. in bulk cobalt metal. The cobalt atom's interaction with a coronene molecule has significantly reduced the spin magnetic moment of cobalt from its free atomic state. Finally, since the ground state of neutral $\mathrm{Co}_{2}$ (coronene) is a quintet, its spin magnetic moment is $4 \mu_{B}$ or $2 \mu_{B}$ per cobalt atom. This is the same value that we determined in our previous study $^{24}$ of $\mathrm{Co}_{2}$ (pyridine). In a very recent experimental study $^{25}$ of benzene-capped cobalt clusters, $\mathrm{Co}_{n}-\left(\mathrm{C}_{6} \mathrm{D}_{6}\right)_{m}$, the magnetic moment per Co atom was reported as greater than $1.6 \mu_{B} /$ at. for $n \leqslant 4$. It is found that the ferromagnetic coupling between the cobalt atoms dominates all the isomers considered in this study, irrespective of the orientation and position of cobalt atoms with the coronene molecule. In the ground state geometry of $\mathrm{Co}_{2}$ (coronene), each of the cobalt atoms has moments of $2.26 \mu_{B}$ and $1.87 \mu_{B}$, while the coronene molecule carries a small negative polarization contributing $-0.13 \mu_{B}$ to the total moment.

We also observe some interesting patterns in the photoelectron spectrum of $\left[\mathrm{Co}_{2} \text { (coronene) }\right]^{-}$. In particular, one observes three peaks in the mid-range of the spectrum, followed by a broad unstructured feature at the high EBE end of the spectrum. This same pattern was observed in our previously reported photoelectron spectra of $\left[\mathrm{Co}_{2}\right.$ (pyridine $\left.)\right]^{-}$ (Ref. 24) and $\left[\mathrm{Co}_{2} \text { (benzene) }\right]^{-5}$. Comparison of the peak positions of various $\left[\mathrm{Co}_{2}\right.$ (organic) $]$ and $\mathrm{Co}$ anions is shown in Table III. This pattern shows little resemblance to the $\mathrm{Co}_{2}^{-}$ spectral pattern; instead, it is interesting to compare these spectra with $\mathrm{Co}^{-}$spectrum. The first three peaks of $\left[\mathrm{Co}_{2} \text { (organic) }\right]^{-}$spectra have a similar splitting as the three peaks in $\mathrm{Co}^{-}$spectrum, but shift to the higher binding energy side. This implies that the $\left[\mathrm{Co}_{2} \text { (organic) }\right]^{-}$clusters have a similar character as the Co atomic anion instead of Co dimer implying that the two Co atoms are not equivalent and one of the Co atoms has a greater excess electron distribution surrounding it than the other, giving rise to the $\mathrm{Co}^{-}$characteristic signature. In other words, $\mathrm{Co}$ (organic) is acting as a solvent for Co anion. This is consistent with our calculation on $\mathrm{Co}_{2}$ (Coronene) $)^{-}$that shows only one Co interacting with coronene, and is also consistent with the calculated charge distribution in the ground state of $\left[\mathrm{Co}_{2} \text { (coronene) }\right]^{-}$[Fig. 4(a)], where the proximal cobalt has a Mulliken charge of $+0.04 e$ and the distal cobalt a charge of $-0.47 e$. 
TABLE III. Experimentally observed vertical detachment energies in $\mathrm{Co}^{-}$and $\mathrm{Co}_{2}$ (organic) ${ }^{-}$spectra, and the observed peak shifts of $\mathrm{Co}_{2}$ (organic) $)^{-}$relative to $\mathrm{Co}^{-}$.

\begin{tabular}{cccccc}
\hline \hline & $\begin{array}{c}\text { Peak 1 } \\
(\mathrm{eV})\end{array}$ & $\begin{array}{c}\text { Peak 2 } \\
(\mathrm{eV})\end{array}$ & $\begin{array}{c}\text { Peak 3 } \\
(\mathrm{eV})\end{array}$ & $\begin{array}{c}\text { Peak } 4 \\
(\mathrm{eV})\end{array}$ & $\begin{array}{c}\text { Shift compared to } \\
\mathrm{Co}^{-}(\mathrm{eV})\end{array}$ \\
\hline $\mathrm{Co}^{-}$ & $\begin{array}{c}0.74 \\
(0.69,0.78)\end{array}$ & 1.09 & $\begin{array}{c}1.55 \\
(1.50,1.59)\end{array}$ & $\ldots$ & 0 \\
{$\left[\mathrm{Co}_{2} \text { (pyridine) }\right]^{-}$} & 0.95 & 1.38 & 1.78 & 2.52 & $\sim+0.2$ \\
{$\left[\mathrm{Co}_{2} \text { (coronene) }\right]^{-}$} & 1.28 & 1.65 & 2.1 & 2.85 & $\sim+0.55$ \\
{$\left[\mathrm{Co}_{2} \text { (benzene) }\right]^{-}$} & 1.43 & 1.79 & 2.25 & 3.05 & $\sim+0.7$ \\
\hline \hline
\end{tabular}

The peak shifts of $\left[\mathrm{Co}_{2} \text { (organic) }\right]^{-}$compared to $\mathrm{Co}$ atomic anion vary with different organic ligands: $\left[\mathrm{Co}_{2} \text { (pyridine) }\right]^{-}$shifts the peaks by an average $0.2 \mathrm{eV}$ higher, $\left[\mathrm{Co}_{2} \text { (coronene) }\right]^{-}$shifts by $0.55 \mathrm{eV}$, while the $\left[\mathrm{Co}_{2} \text { (benzene) }\right]^{-}$the most, by $0.7 \mathrm{eV}$ higher (Table III). This implies that $[\mathrm{Co}($ benzene $)]$ stabilizes $\mathrm{Co}^{-}$the most, $[\mathrm{Co}($ coronene $)]$ slightly less, while $[\mathrm{Co}$ (pyridine) $]$ the least. Comparable shifts for $\left[\mathrm{Co}_{2}\right.$ (coronene) $]$ and $\left[\mathrm{Co}_{2}\right.$ (benzene) $]$ come as no surprise due to a similarly aromatic nature of the two organic ligands involved.

\section{SUMMARY AND CONCLUSIONS}

$[\mathrm{Co}(\text { coronene })]^{-}$and $\left[\mathrm{Co}_{2}\right.$ (coronene $\left.)\right]^{-}$clusters were produced in a laser vaporization source. Density functional theory and photoelectron spectroscopy experiments have been used to study the equilibrium geometries, electronic structure, and magnetic properties of $\mathrm{Co}$ atom and Co dimer supported on a coronene molecule. Comparison of calculated VDEs and AEAs of $\mathrm{Co}_{m}$ (coronene $)^{-}(m=1,2)$ with our experimental observations showed good agreement, thereby allowing us to make conclusions about the structures of anionic and neutral complexes.

In contrast to the previously reported ${ }^{16} \mathrm{Fe}_{m}$ (coronene) $(m=1,2)$ complexes, where the metal atoms preferred $\eta^{6}$ type of bond interactions, in $\mathrm{Co}_{m}$ (coronene) $(m=1,2)$ the cobalt atoms always prefer to minimize the number of $\mathrm{Co}$ coronene bond interactions. In neutral Co(coronene), there is a competition between the $\eta^{6}$ and $\eta^{2}$ binding sites, while in the anion, cobalt preferred to interact with only two carbon atoms of the coronene molecule.

For $\mathrm{Co}_{2}$ (coronene), both anion and neutral, the $\eta^{6}$ binding site on the coronene molecule is not a favorable binding site for Co dimer, but it prefers $\eta^{2}$ bonded structures. Theoretically, two different structural isomers were found to compete in stabilizing the anion and neutral $\mathrm{Co}_{2}$ (coronene) complexes. In both of these structures the metal atoms prefer $\eta^{2}$ binding sites. However, a comparison of theoretical results with the experimental observations has allowed us to identify the majority structural isomer of anionic $\left[\mathrm{Co}_{2} \text { (coronene) }\right]^{-}$ complex as the isomer with metal dimer bound to a peripheral ring's $\eta^{2}$ site via a single metal atom [Fig. 4(a)].

The magnetic properties of the $\mathrm{Co}_{m}$ (coronene) complexes are studied by optimizing the total spin multiplicities of these complexes and determining the distribution of the total magnetic moments. We find that while the magnetic moment of the Co atom in $\mathrm{Co}$ (coronene) complex is $1 \mu_{B}$, the total magnetic moment of $\mathrm{Co}_{2}$ (coronene) complex is $4 \mu_{B}$. In
$\mathrm{Co}_{2}$ (coronene) complex there is a ferromagnetic coupling between the Co atoms where the Co atoms carry magnetic moments of $2.26 \mu_{B}$ and $1.87 \mu_{B}$ individually.

It is intriguing to find that the ground state geometries of the most preferred binding sites on coronene are entirely different for the $\mathrm{Co}_{2}$ (coronene) and the previously reported $\mathrm{Fe}_{2}$ (coronene) systems. It seems that transition metalcoronene complexes can exhibit rich structural and bonding variations depending on the charge and transition metal atom. Hence, it would be interesting to further explore the bonding, ground state geometries, and magnetic properties of the other $3 d$-metal atoms and clusters interacting with coronene. Both experimental and theoretical efforts in this direction are in progress.

\section{ACKNOWLEDGMENTS}

One of the authors (K.B.) thanks the Division of Materials Sciences and Engineering, Basic Energy Sciences, U.S. Department of Energy for support of this work under Grant No. DE-FG02-95ER45538. Acknowledgement is also made to the Donors of The Petroleum Research Fund, administered by The American Chemical Society, for partial support of this research (Grant No. 28452-AC6). Two of the authors (A.K.K. and P.J.) acknowledge support from the U.S. Department of Energy (DEFG01-96ER45579).

${ }^{1}$ T. Kurikawa, H. Takeda, M. Hirano, K. Judai, T. Arita, S. Nagao, A. Nakajima, and K. Kaya, Organometallics 18, 1430 (1999).

${ }^{2}$ K. Hoshino, T. Kurikawa, H. Takeda, A. Nakajima, and K. Kaya, J. Phys. Chem. 99, 3053 (1995).

${ }^{3}$ P. Weis, P. R. Kemper, and M. T. Bowers, J. Phys. Chem. A 101, 8207 (1997)

${ }^{4}$ T. Yasuike, A. Nakajima, S. Yabushita, and K. Kaya, J. Phys. Chem. A 101, 5360 (1997)

${ }^{5}$ M. Gerhards, O. C. Thomas, J. M. Nilles, W.-J. Zheng, and K. H. Bowen, J. Chem. Phys. 116, 10247 (2002).

${ }^{6}$ W.-J. Zheng, J. M. Nilles, O. C. Thomas, and K. H. Bowen, Chem. Phys. Lett. 401, 266 (2005)

${ }^{7}$ W.-J. Zheng, J. M. Nilles, O. C. Thomas, and K. H. Bowen, J. Chem. Phys. 122, 044306 (2005).

${ }^{8}$ B. K. Rao and P. Jena, J. Chem. Phys. 116, 1343 (2002); 117, 5234 (2002)

${ }^{9}$ A. K. Kandalam, B. K. Rao, P. Jena, and R. Pandey, J. Chem. Phys. 120, 10414 (2004) and references therein.

${ }^{10}$ L. J. Allamandola, A. G. G. M. Tielens, and R. J. Barker, Astrophys. J. 290, L25 (1985); D. K. Bohme, Chem. Rev. (Washington, D.C.) 92 , 1487 (1992).

${ }^{11}$ B. P. Pozniak and R. C. Dunbar, J. Am. Chem. Soc. 119, 10439 (1997).

${ }^{12}$ J. W. Buchanan, J. E. Reddic, G. A. Grieves, and M. A. Duncan, J. Phys. Chem. A 102, 6390 (1998).

${ }^{13}$ N. R. Foster, G. A. Grieves, J. W. Buchanan, N. D. Flynn, and M. A. Duncan, J. Phys. Chem. A 104, 11055 (2000).

${ }^{14}$ M. A. Duncan, A. M. Knight, Y. Negishi, S. Nagao, K. Judai, A. Naka- 
jima, and K. Kaya, J. Phys. Chem. A 105, 10093 (2001).

${ }^{15}$ R. C. Dunbar, J. Phys. Chem. A 106, 9809 (2002).

${ }^{16}$ L. Senapati, S. K. Nayak, B. K. Rao, and P. Jena, J. Chem. Phys. 118, 8671 (2003).

${ }^{17}$ M. J. Frisch, G. W. Trucks, H. B. Schlegel et al., GAUSSIAN 03, Revision C.02, Gaussian, Inc., Wallingford, CT, 2004.

${ }^{18}$ A. D. Becke, Phys. Rev. A 38, 3098 (1988).

${ }^{19}$ J. P. Perdew and Y. Wang, Phys. Rev. B 45, 13244 (1992).

${ }^{20}$ D. J. Tozer and N. C. Handy, J. Chem. Phys. 109, 10180 (1998).
${ }^{21}$ M. A. Duncan, A. M. Knight, Y. Negishi, S. Nagao, Y. Nakamura, A. Kato, A. Nakajima, and K. Kaya, Chem. Phys. Lett. 309, 49 (1999).

${ }^{22}$ G. Chen, R. G. Cooks, E. Corpuz, and L. T. Scott, J. Am. Soc. Mass Spectrom. 7, 619 (1996).

${ }^{23}$ R. R. Corderman, P. C. Engelking, and W. C. Lineberger, J. Chem. Phys. 70, 4474 (1979).

${ }^{24}$ B. D. Edmonds, A. K. Kandalam, S. N. Khanna, X. Li, A. Grubisic, I. Khanna, and K. H. Bowen, J. Chem. Phys. 124, 074316 (2006).

${ }^{25}$ M. B. Knickelbein, J. Chem. Phys. 125, 044308 (2006). 